\title{
APPENDIX: Health Care Usage and Health Status of Immigrant Children: The Effects of Nativity versus Citizenship
}

\author{
Mónica, García-Pérez *
}

October 2, 2015

Figure 1: Children's BMI density by Region of Birth and Race/Ethnicity

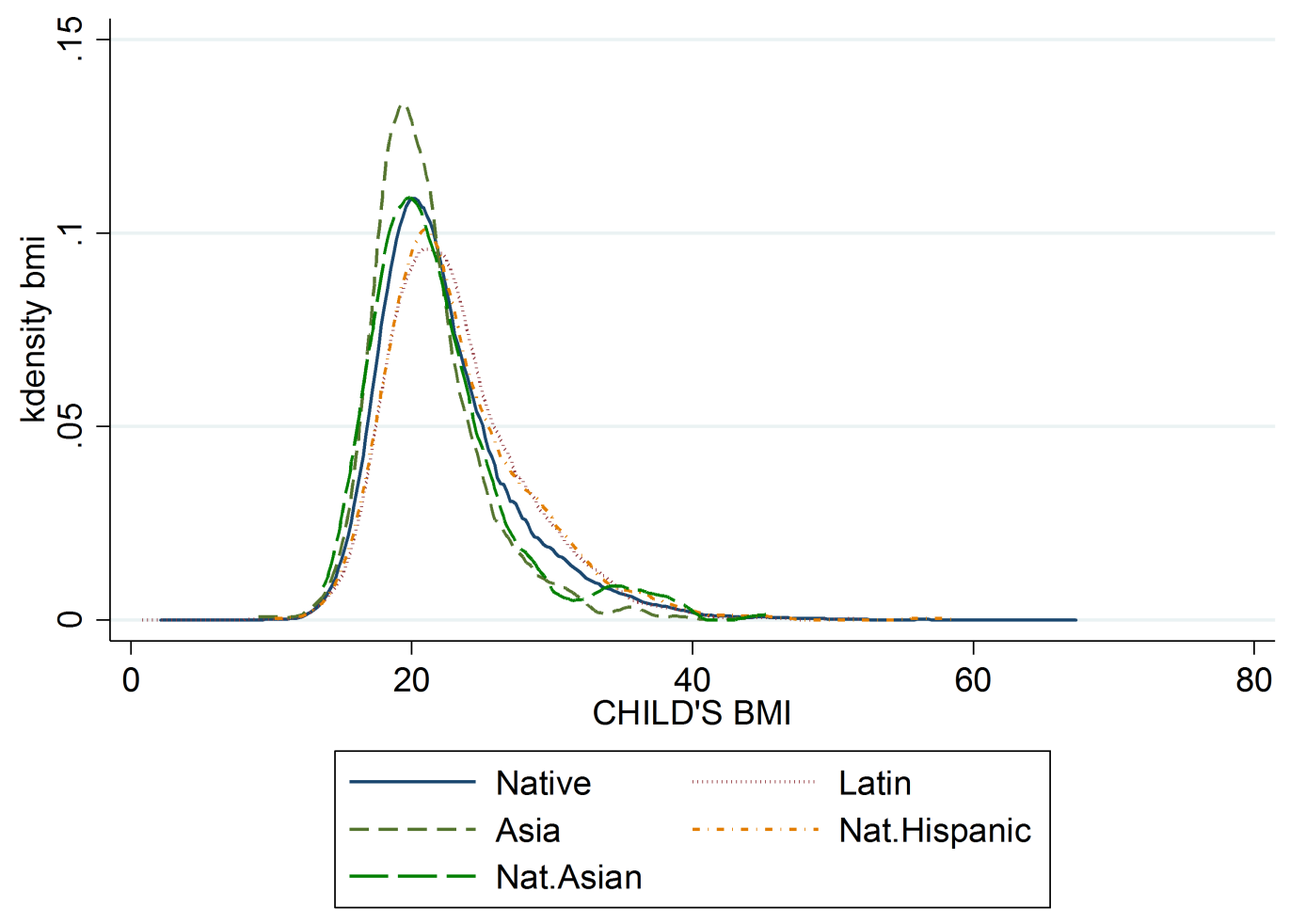

Note:Complex sample design considered.

*García-Pérez: St. Cloud State University, SH-386, 4th Ave. S St. Cloud, MN 56301, migarciaperez@stcloudstate.edu. Thank to comments received at the 2013 ASSA meetings from discussant and attendants. All comments are responsibility of the author. 
Figure 2: Doctor Visits: Children of Immigrants by Maternal Region of Birth and Years in the US

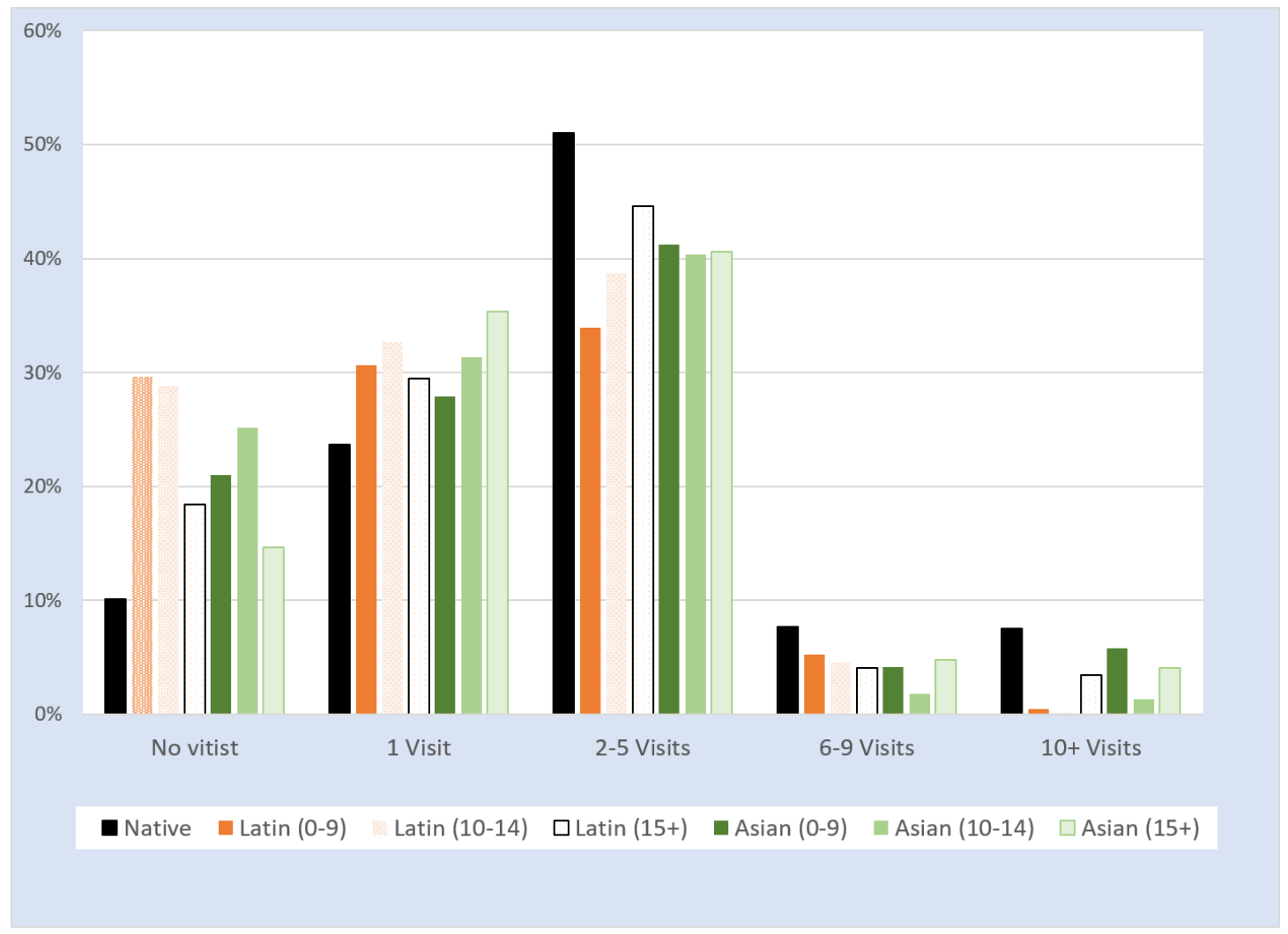

Note:Complex sample design considered. 
Table 1: Summary Statistics - Weighted

\begin{tabular}{|c|c|c|c|c|c|}
\hline & Natives & Nat. Hispanic & Nat. Asian & Latin American & Asia \\
\hline Obese & $0.150^{* * *}$ & $0.206^{* * *}$ & $0.109^{* * *}$ & $0.191^{* * *}$ & $0.0790^{* * *}$ \\
\hline \multirow{3}{*}{ 0-9 Yrs in the US } & $(0.004)$ & $(0.013)$ & $(0.027)$ & $(0.007)$ & $(0.011)$ \\
\hline & & & & $0.0390^{* * *}$ & $0.0714^{* * *}$ \\
\hline & & & & $(0.004)$ & $(0.009)$ \\
\hline \multirow[t]{2}{*}{$10-14$ Yrs in the US } & & & & $0.0841^{* * *}$ & $0.105^{* * *}$ \\
\hline & & & & $(0.006)$ & $(0.013)$ \\
\hline $15+$ Yrs in the US & & & & $\begin{array}{r}0.817^{* * *} \\
(0.008)\end{array}$ & $\begin{array}{r}0.823^{* * *} \\
(0.015)\end{array}$ \\
\hline \multirow[t]{2}{*}{ No visits } & $0.101^{* * *}$ & $0.137^{* * *}$ & $0.138^{* * *}$ & $0.195^{* * *}$ & $0.162^{* * *}$ \\
\hline & $(0.003)$ & $(0.011)$ & $(0.031)$ & $(0.009)$ & $(0.014)$ \\
\hline \multirow[t]{2}{*}{1 Visit } & $0.237^{* * * *}$ & $0.245^{* * *}$ & $0.233^{* * *}$ & $0.295^{* * *}$ & $0.349^{* * *}$ \\
\hline & $(0.004)$ & $(0.013)$ & $(0.039)$ & $(0.010)$ & $(0.017)$ \\
\hline \multirow[t]{2}{*}{$2-5$ visits } & $0.510^{* * *}$ & $0.503^{* * *}$ & $0.495^{* * *}$ & $0.434^{* * *}$ & $0.406^{* * *}$ \\
\hline & $(0.005)$ & $(0.015)$ & $(0.053)$ & $(0.009)$ & $(0.020)$ \\
\hline \multirow[t]{2}{*}{$6-9$ visits } & $0.0770^{* * *}$ & $0.0627^{* * *}$ & $0.0674^{* *}$ & $0.0430^{* * *}$ & $0.0441^{* * *}$ \\
\hline & $(0.003)$ & $(0.007)$ & $(0.025)$ & $(0.004)$ & $(0.010)$ \\
\hline \multirow[t]{2}{*}{$10+$ visits } & $0.0751^{* * *}$ & $0.0519^{* * *}$ & $0.0663^{* * *}$ & $0.0337^{* * *}$ & $0.0391^{* * *}$ \\
\hline & $(0.003)$ & $(0.007)$ & $(0.022)$ & $(0.004)$ & $(0.008)$ \\
\hline \multirow[t]{2}{*}{ Child: Female } & $0.491^{* * *}$ & $0.495^{* * *}$ & $0.501 * * *$ & $0.497^{* * *}$ & $0.479^{* * *}$ \\
\hline & $(0.005)$ & $(0.014)$ & $(0.051)$ & $(0.010)$ & $(0.020)$ \\
\hline \multirow[t]{2}{*}{ Child: Age } & $14.47^{* * *}$ & $14.42^{* * *}$ & $14.42^{* * *}$ & $14.49^{* * *}$ & $14.54^{* * *}$ \\
\hline & $(0.017)$ & $(0.050)$ & $(0.160)$ & $(0.033)$ & $(0.069)$ \\
\hline \multirow[t]{2}{*}{ Child: Foreign born } & $0.0111^{* * *}$ & $0.0103^{* * *}$ & $0.0470^{* *}$ & $0.238^{* * *}$ & $0.275^{* * *}$ \\
\hline & $(0.001)$ & $(0.003)$ & $(0.018)$ & $(0.009)$ & $(0.018)$ \\
\hline \multirow[t]{2}{*}{ Mother: BMI } & $15.42^{* * *}$ & $15.25^{* * *}$ & $11.14^{* * *}$ & $13.41^{* * *}$ & $9.790^{* * *}$ \\
\hline & $(0.206)$ & $(0.543)$ & $(1.292)$ & $(0.333)$ & $(0.573)$ \\
\hline \multirow[t]{2}{*}{ Mother: Unemployed } & $0.292^{* * *}$ & $0.305^{* * *}$ & $0.295 * * *$ & $0.432^{* * *}$ & $0.322^{* * *}$ \\
\hline & $(0.005)$ & $(0.015)$ & $(0.047)$ & $(0.011)$ & $(0.019)$ \\
\hline \multirow[t]{2}{*}{ Mother: Married } & $0.690^{* * *}$ & $0.605^{* * *}$ & $0.733^{* * *}$ & $0.725^{* * *}$ & $0.863^{* * *}$ \\
\hline & $(0.005)$ & $(0.015)$ & $(0.037)$ & $(0.009)$ & $(0.013)$ \\
\hline \multirow[t]{2}{*}{ Mother: High School Dropout } & $0.0846^{* * * *}$ & $0.158^{* * *}$ & $0.0527^{* *}$ & $0.514^{* * *}$ & $0.131^{* * *}$ \\
\hline & $(0.003)$ & $(0.012)$ & $(0.021)$ & $(0.011)$ & $(0.014)$ \\
\hline \multirow[t]{2}{*}{ Mother: Some college } & $0.265^{* * *}$ & $0.315^{* * *}$ & $0.158^{* * *}$ & $0.146^{* * *}$ & $0.130^{* * *}$ \\
\hline & $(0.005)$ & $(0.014)$ & $(0.031)$ & $(0.007)$ & $(0.014)$ \\
\hline \multirow[t]{2}{*}{ Mother: College Degree } & $0.352^{* * *}$ & $0.243^{* * *}$ & $0.383^{* * *}$ & $0.128^{* * *}$ & $0.426^{* * *}$ \\
\hline & $(0.005)$ & $(0.016)$ & $(0.051)$ & $(0.007)$ & $(0.020)$ \\
\hline \multirow[t]{2}{*}{ Mother: Graduate School } & $0.0972^{* * *}$ & $0.0501^{* * *}$ & $0.136^{* * *}$ & $0.0289^{* * *}$ & $0.147^{* * *}$ \\
\hline & $(0.003)$ & $(0.006)$ & $(0.029)$ & $(0.003)$ & $(0.015)$ \\
\hline Mother: Age & $43.04^{* * *}$ & $39.53^{* * *}$ & $43.11^{* * *}$ & $41.18^{* * *}$ & $43.62^{* * *}$ \\
\hline & $(0.089)$ & $(0.223)$ & $(0.852)$ & $(0.153)$ & $(0.284)$ \\
\hline Below Poverty level & $0.187^{* * *}$ & $0.270^{* * *}$ & $0.150^{* * *}$ & $0.360^{* * *}$ & $0.200^{* * *}$ \\
\hline & $(0.004)$ & $(0.014)$ & $(0.046)$ & $(0.009)$ & $(0.016)$ \\
\hline Above Poverty level & $0.149^{* * *}$ & $0.212^{* * *}$ & $0.134^{* * *}$ & $0.358^{* * *}$ & $0.142^{* * *}$ \\
\hline & $(0.004)$ & $(0.013)$ & $(0.037)$ & $(0.011)$ & $(0.016)$ \\
\hline Mother: Usual Place of Care & $0.553^{* * *}$ & $0.594^{* * *}$ & $0.646^{* * *}$ & $0.651^{* * *}$ & $0.649^{* * *}$ \\
\hline & $(0.005)$ & $(0.016)$ & $(0.047)$ & $(0.010)$ & $(0.020)$ \\
\hline Mother: Uninsured & $0.132^{* * *}$ & $0.181^{* * *}$ & $0.122^{* * *}$ & $0.436^{* * *}$ & $0.134^{* * *}$ \\
\hline & $(0.004)$ & $(0.011)$ & $(0.033)$ & $(0.011)$ & $(0.013)$ \\
\hline Mother: Non citizen & & & & $0.614^{* * *}$ & $0.271^{* * *}$ \\
\hline & & & & $(0.012)$ & $(0.019)$ \\
\hline Good Health & $0.978^{* * *}$ & $0.971^{* * *}$ & $0.966^{* * *}$ & $0.965^{* * *}$ & $0.990^{* * *}$ \\
\hline & $(0.002)$ & $(0.004)$ & $(0.024)$ & $(0.004)$ & $(0.003)$ \\
\hline Survey year & $2,011^{* * *}$ & $2,011^{* * *}$ & $2,011^{* * *}$ & $2,011^{* * *}$ & $2,011^{* * *}$ \\
\hline & $(0.022)$ & $(0.069)$ & $(0.216)$ & $(0.040)$ & $(0.080)$ \\
\hline US region & $2.595^{* * *}$ & $3.062^{* * *}$ & $3.118^{* * *}$ & $3.001^{* * *}$ & $2.950^{* * *}$ \\
\hline & $(0.015)$ & $(0.039)$ & $(0.107)$ & $(0.031)$ & $(0.048)$ \\
\hline Observations & 16,660 & 1,851 & 297 & 4,160 & 1,111 \\
\hline
\end{tabular}


Table 2: Odd Ratios: Logit Regression - Comparison with Children of Native Born Mothers

\begin{tabular}{|c|c|c|c|c|}
\hline & (1) & (2) & (3) & (4) \\
\hline VARIABIFS & Variables & Nat_Hicn/I atin & Nat/Asia & Nat-Asian / 1 si \\
\hline \multirow{2}{*}{$0-9$ Yrs in the US } & $\begin{array}{l}\text { Nat/Latin } \\
0.963\end{array}$ & $\begin{array}{c}\text { Nat-Hisp/Latın } \\
0.917\end{array}$ & $\begin{array}{c}\text { Nat/Asia } \\
0.524\end{array}$ & $\begin{array}{c}\text { Nat-Asian/Asia } \\
0.733\end{array}$ \\
\hline & $(0.346)$ & $(0.336)$ & $(0.397)$ & $(0.581)$ \\
\hline \multirow[t]{2}{*}{$10-14$ Yrs in the US } & $0.692^{*}$ & $0.662^{*}$ & $0.107^{* * * *}$ & $0.131^{* *}$ \\
\hline & $(0.154)$ & $(0.153)$ & $(0.0817)$ & $(0.107)$ \\
\hline \multirow[t]{2}{*}{$15+$ Yrs in the US } & $1.113^{*}$ & $0.840^{*}$ & & \\
\hline & $(0.0718)$ & $(0.0838)$ & & \\
\hline \multirow[t]{2}{*}{ Child: Female } & $0.622^{* * *}$ & $0.620^{* * *}$ & $0.629^{* * *}$ & $0.466^{* * *}$ \\
\hline & $(0.0295)$ & $(0.0505)$ & $(0.0346)$ & $(0.110)$ \\
\hline \multirow[t]{2}{*}{ CHild: age } & $0.911^{* * *}$ & $0.855^{* * *}$ & $0.927^{* * * *}$ & 0.980 \\
\hline & $(0.0139)$ & $(0.0206)$ & $(0.0160)$ & $(0.0580)$ \\
\hline \multirow[t]{2}{*}{ Child:foreign } & 0.860 & 0.948 & $0.611^{* *}$ & $0.422^{* *}$ \\
\hline & $(0.118)$ & $(0.148)$ & $(0.148)$ & $(0.177)$ \\
\hline \multirow[t]{2}{*}{ Mother: bmi } & $1.013^{* * *}$ & $1.014^{* * *}$ & $1.014^{* * *}$ & 1.000 \\
\hline & $(0.00157)$ & $(0.00318)$ & $(0.00170)$ & $(0.0113)$ \\
\hline \multirow[t]{2}{*}{ Mother: unemployed } & 1.061 & $1.281^{* * *}$ & 1.011 & 0.998 \\
\hline & $(0.0565)$ & $(0.101)$ & $(0.0611)$ & $(0.236)$ \\
\hline \multirow[t]{2}{*}{ Mother: married } & $0.756^{* * *}$ & 0.934 & $0.713^{* * *}$ & 0.655 \\
\hline & $(0.0383)$ & $(0.0818)$ & $(0.0413)$ & $(0.193)$ \\
\hline \multirow[t]{2}{*}{ Mother: High School Dropout } & 1.114 & 1.130 & 1.126 & 1.686 \\
\hline & $(0.0864)$ & $(0.132)$ & $(0.110)$ & $(0.612)$ \\
\hline \multirow[t]{2}{*}{ esce_h } & 0.938 & 0.962 & 0.925 & 0.582 \\
\hline & $(0.0710)$ & $(0.135)$ & $(0.0750)$ & $(0.198)$ \\
\hline \multirow[t]{2}{*}{ eced_h } & $0.637^{* * *}$ & 0.865 & $0.625^{* * *}$ & $0.437^{* * *}$ \\
\hline & $(0.0461)$ & $(0.124)$ & $(0.0483)$ & $(0.123)$ \\
\hline \multirow[t]{2}{*}{ egse_h } & $0.438^{* * *}$ & $0.393^{* * *}$ & $0.458^{* * *}$ & $0.458^{*}$ \\
\hline & $(0.0574)$ & $(0.121)$ & $(0.0618)$ & $(0.207)$ \\
\hline \multirow[t]{2}{*}{ age_h } & 1.001 & 1.003 & 0.999 & 0.976 \\
\hline & $(0.00283)$ & $(0.00498)$ & $(0.00311)$ & $(0.0151)$ \\
\hline \multirow[t]{2}{*}{ nusualpl_h } & $1.397^{* * *}$ & 1.140 & $1.400^{* * *}$ & 1.006 \\
\hline & $(0.0904)$ & $(0.130)$ & $(0.0995)$ & $(0.377)$ \\
\hline \multirow[t]{2}{*}{ nocoverage_h } & $1.177^{* *}$ & 1.127 & $1.173^{*}$ & $0.550^{* *}$ \\
\hline & $(0.0850)$ & $(0.113)$ & $(0.0980)$ & $(0.165)$ \\
\hline \multirow[t]{2}{*}{ goodhealth } & $0.441^{* * *}$ & $0.553^{* * *}$ & $0.406^{* * *}$ & 1.189 \\
\hline & $(0.0584)$ & $(0.107)$ & $(0.0619)$ & $(0.769)$ \\
\hline \multirow[t]{2}{*}{ year } & 0.991 & 0.981 & 0.996 & 1.033 \\
\hline & $(0.0115)$ & $(0.0209)$ & $(0.0133)$ & $(0.0543)$ \\
\hline \multirow[t]{2}{*}{ region } & 1.007 & 1.027 & 0.991 & 0.869 \\
\hline & $(0.0261)$ & $(0.0396)$ & $(0.0290)$ & $(0.0993)$ \\
\hline \multirow[t]{2}{*}{ 4.regionb } & & & $0.720^{*}$ & $0.624^{*}$ \\
\hline & & & $(0.121)$ & $(0.172)$ \\
\hline Constant & $\begin{array}{c}1.412 \mathrm{e}+08 \\
(3.304 \mathrm{e}+09)\end{array}$ & $\begin{array}{c}4.326 \mathrm{e}+17 \\
(1.856 \mathrm{e}+19)\end{array}$ & $\begin{array}{c}4,455 \\
(119,554)\end{array}$ & $\begin{array}{c}0 \\
(0)\end{array}$ \\
\hline Observations & 20,828 & 6,019 & 17,771 & 1,525 \\
\hline
\end{tabular}

Note: Observations: 22,638. Other controls include: year, US region, maternal education level, family size, single parent, parental employment status. Reference group depends on each column. Those compared to Natives, male native children with US born parents, with health insurance and usual place of care is the reference group. When compared to Nat. Hispanic/Asian: male native children with US born Hispanic/Asian parents, with health insurance and usual place of care is the reference group (OD=1.00) Standard errors in parenthesis. ${ }^{* *} \mathrm{p}_{\mathrm{i}} 0.01,{ }^{* *} \mathrm{p}_{\mathrm{i}} 0.05,{ }^{*} \mathrm{p}_{\mathrm{i}} 0.1$ [Source] Author's calculations. 


\section{Decomposition Analysis - Comparison with Children of Native Born Mothers}

\begin{tabular}{|c|c|c|c|c|c|}
\hline & Variables & Nat-Latin & Nat-Asia & Nat/Hisp-Latin & Nat/Asian-Asia \\
\hline \multirow[t]{5}{*}{ overall } & group_1 & $\begin{array}{c}0.150 \\
{[0.004]^{* *}}\end{array}$ & $\begin{array}{c}0.150 \\
{[0.004]^{* *}}\end{array}$ & $\begin{array}{c}0.206 \\
{[0.013]^{* *}}\end{array}$ & $\begin{array}{c}0.163 \\
{[0.023]^{* *}}\end{array}$ \\
\hline & group_2 & $\begin{array}{c}0.191 \\
{[0.007]^{* *}}\end{array}$ & $\begin{array}{c}0.079 \\
{[0.011]^{* *}}\end{array}$ & $\begin{array}{c}0.191 \\
{[0.007]^{* *}}\end{array}$ & $\begin{array}{c}0.079 \\
{[0.011]^{* *}}\end{array}$ \\
\hline & difference & $\begin{array}{c}-0.041 \\
{[0.008]^{* *}}\end{array}$ & $\begin{array}{c}0.071 \\
{[0.012]^{* *}}\end{array}$ & $\begin{array}{c}0.016 \\
{[0.014]}\end{array}$ & $\begin{array}{c}0.084 \\
{[0.026]^{* *}}\end{array}$ \\
\hline & explained & $\begin{array}{c}-0.026 \\
{[0.006]^{* *}}\end{array}$ & $\begin{array}{c}0.044 \\
{[0.005]^{* *}}\end{array}$ & $\begin{array}{c}-0.011 \\
{[0.009]}\end{array}$ & $\begin{array}{c}0.044 \\
{[0.014]^{* *}}\end{array}$ \\
\hline & unexplained & $\begin{array}{c}-0.015 \\
{[0.009]+}\end{array}$ & $\begin{array}{c}0.026 \\
{[0.012]^{*}}\end{array}$ & $\begin{array}{c}0.027 \\
{[0.016]+}\end{array}$ & $\begin{array}{c}0.041 \\
{[0.024]+}\end{array}$ \\
\hline \multirow[t]{18}{*}{ explained } & yrsinusrec $\_h==1.0000$ & $\begin{array}{c}0.000 \\
{[0.002]}\end{array}$ & $\begin{array}{c}0.003 \\
{[0.004]}\end{array}$ & $\begin{array}{c}0.001 \\
{[0.002]}\end{array}$ & $\begin{array}{c}0.002 \\
{[0.004]}\end{array}$ \\
\hline & yrsinusrec_h $h==2.0000$ & $\begin{array}{c}0.004 \\
{[0.002]}\end{array}$ & $\begin{array}{c}0.018 \\
{[0.005]^{* *}}\end{array}$ & $\begin{array}{c}0.006 \\
{[0.003]+}\end{array}$ & $\begin{array}{c}0.016 \\
{[0.006]^{* *}}\end{array}$ \\
\hline & female_c & $\begin{array}{c}0.000 \\
{[0.001]}\end{array}$ & $\begin{array}{c}-0.000 \\
{[0.001]}\end{array}$ & $\begin{array}{c}0.000 \\
{[0.001]}\end{array}$ & $\begin{array}{c}-0.002 \\
{[0.002]}\end{array}$ \\
\hline & Age & $\begin{array}{c}0.000 \\
{[0.000]}\end{array}$ & $\begin{array}{c}0.000 \\
{[0.000]}\end{array}$ & $\begin{array}{c}0.002 \\
{[0.002]}\end{array}$ & $\begin{array}{c}0.000 \\
{[0.001]}\end{array}$ \\
\hline & foreign_c & $\begin{array}{c}0.004 \\
{[0.004]}\end{array}$ & $\begin{array}{c}0.010 \\
{[0.005]^{*}}\end{array}$ & $\begin{array}{c}0.002 \\
{[0.006]}\end{array}$ & $\begin{array}{c}0.017 \\
{[0.009]+}\end{array}$ \\
\hline & BMI mother & $\begin{array}{c}0.003 \\
{[0.001]^{* *}}\end{array}$ & $\begin{array}{c}0.006 \\
{[0.001]^{* *}}\end{array}$ & $\begin{array}{c}0.004 \\
{[0.002]^{*}}\end{array}$ & $\begin{array}{c}0.000 \\
{[0.005]}\end{array}$ \\
\hline & unemployed_h & $\begin{array}{c}-0.001 \\
{[0.001]}\end{array}$ & $\begin{array}{c}-0.000 \\
{[0.000]}\end{array}$ & $\begin{array}{c}-0.005 \\
{[0.002]^{* *}}\end{array}$ & $\begin{array}{c}-0.000 \\
{[0.001]}\end{array}$ \\
\hline & married & $\begin{array}{c}0.001 \\
{[0.000]^{* *}}\end{array}$ & $\begin{array}{c}0.004 \\
{[0.001]^{* *}}\end{array}$ & $\begin{array}{c}0.001 \\
{[0.002]}\end{array}$ & $\begin{array}{c}0.008 \\
{[0.006]}\end{array}$ \\
\hline & High school drop out Mother & $\begin{array}{c}-0.006 \\
{[0.004]}\end{array}$ & $\begin{array}{c}-0.000 \\
{[0.000]}\end{array}$ & $\begin{array}{c}-0.007 \\
{[0.007]}\end{array}$ & $\begin{array}{c}-0.000 \\
{[0.001]}\end{array}$ \\
\hline & Some college education Mother & $\begin{array}{c}-0.001 \\
{[0.001]}\end{array}$ & $\begin{array}{c}-0.001 \\
{[0.001]}\end{array}$ & $\begin{array}{c}-0.001 \\
{[0.004]}\end{array}$ & $\begin{array}{c}-0.005 \\
{[0.004]}\end{array}$ \\
\hline & College education degree Mother & $\begin{array}{c}-0.013 \\
{[0.002]^{* *}}\end{array}$ & $\begin{array}{c}0.003 \\
{[0.001]^{* *}}\end{array}$ & $\begin{array}{c}-0.003 \\
{[0.003]}\end{array}$ & $\begin{array}{c}0.007 \\
{[0.004]+}\end{array}$ \\
\hline & Graduate School education Mother & $\begin{array}{c}-0.007 \\
{[0.001]^{* *}}\end{array}$ & $\begin{array}{c}0.003 \\
{[0.001]^{* *}}\end{array}$ & $\begin{array}{c}-0.003 \\
{[0.002]^{*}}\end{array}$ & $\begin{array}{c}0.004 \\
{[0.003]}\end{array}$ \\
\hline & age_h & $\begin{array}{c}0.000 \\
{[0.001]}\end{array}$ & $\begin{array}{c}0.000 \\
{[0.000]}\end{array}$ & $\begin{array}{c}-0.001 \\
{[0.001]}\end{array}$ & $\begin{array}{c}0.003 \\
{[0.003]}\end{array}$ \\
\hline & nusualpl h & $\begin{array}{c}-0.004 \\
{[0.001]^{* *}}\end{array}$ & $\begin{array}{c}-0.002 \\
{[0.001]^{* *}}\end{array}$ & $\begin{array}{c}-0.001 \\
{[0.001]}\end{array}$ & $\begin{array}{c}-0.000 \\
{[0.003]}\end{array}$ \\
\hline & nocoverage $\_h$ & $\begin{array}{c}-0.006 \\
{[0.003]^{*}}\end{array}$ & $\begin{array}{l}-0.000 \\
{[0.000]}\end{array}$ & $\begin{array}{c}-0.005 \\
{[0.004]}\end{array}$ & $\begin{array}{c}-0.002 \\
{[0.002]}\end{array}$ \\
\hline & goodhealth & $\begin{array}{c}-0.001 \\
{[0.000]^{* *}}\end{array}$ & $\begin{array}{c}0.001 \\
{[0.000]^{* *}}\end{array}$ & $\begin{array}{c}-0.001 \\
{[0.001]}\end{array}$ & $\begin{array}{c}-0.000 \\
{[0.002]}\end{array}$ \\
\hline & Survey year & $\begin{array}{c}0.000 \\
{[0.000]}\end{array}$ & $\begin{array}{c}0.000 \\
{[0.000]}\end{array}$ & $\begin{array}{c}0.000 \\
{[0.000]}\end{array}$ & $\begin{array}{c}-0.001 \\
{[0.001]}\end{array}$ \\
\hline & Region of residence & $\begin{array}{c}-0.000 \\
{[0.001]}\end{array}$ & $\begin{array}{c}0.000 \\
{[0.001]}\end{array}$ & $\begin{array}{c}0.000 \\
{[0.000]}\end{array}$ & $\begin{array}{c}-0.003 \\
{[0.003]}\end{array}$ \\
\hline
\end{tabular}

Observations: 22,638. Other controls include: year, US region, maternal education level, family size, single parent, parental employment status. Reference group depends on each column. Those compared to Natives, male native children with US born parents, with health insurance and usual place of care is the reference group. When compared to Nat. Hispanic/Asian: male native children with US born Hispanic/Asian parents, with health insurance and usual place of care is the reference group (OD=1.00). Standard errors in parenthesis. $+p<0.1 ;^{*} p<0.05 ;{ }^{* *} p<0.01$ 


\section{Percent changes: Poisson - Comparison with Children of Native Born}

\begin{tabular}{|c|c|c|c|c|}
\hline Variables & Nat-Latin & Nat.Hispanic-Latin & Nat-Asia & Nat.Asian-Asia \\
\hline \multirow[t]{2}{*}{ regionb } & -0.099 & -0.041 & -0.043 & -0.031 \\
\hline & {$[0.018]^{* *}$} & {$[0.008]^{* *}$} & {$[0.021]^{*}$} & {$[0.016]+$} \\
\hline \multirow[t]{2}{*}{ yrsinusrec_h $h==1.0000$} & -0.179 & 0.019 & -0.158 & 0.102 \\
\hline & {$[0.076]^{*}$} & [0.109] & {$[0.079]^{*}$} & {$[0.121]$} \\
\hline \multirow[t]{2}{*}{ yrsinusrec_h $==2.0000$} & -0.165 & -0.165 & -0.151 & -0.094 \\
\hline & {$[0.063]^{* *}$} & {$[0.118]$} & {$[0.065]^{*}$} & {$[0.118]$} \\
\hline \multirow[t]{2}{*}{ female_c } & 0.061 & 0.056 & 0.078 & 0.025 \\
\hline & {$[0.010]^{* *}$} & {$[0.011]^{* *}$} & {$[0.022]^{* *}$} & {$[0.045]$} \\
\hline \multirow[t]{2}{*}{ Age } & 0.004 & 0.006 & -0.011 & 0.008 \\
\hline & [0.003] & {$[0.003]+$} & {$[0.006]+$} & {$[0.014]$} \\
\hline \multirow[t]{2}{*}{ foreign_c } & -0.079 & -0.026 & -0.104 & -0.043 \\
\hline & {$[0.031]^{*}$} & {$[0.042]$} & {$[0.038]^{* *}$} & {$[0.074]$} \\
\hline \multirow[t]{2}{*}{ BMI mother } & -0.001 & -0.001 & 0.000 & -0.001 \\
\hline & {$[0.000]$} & {$[0.000]$} & {$[0.001]$} & {$[0.003]$} \\
\hline \multirow[t]{2}{*}{ obese } & 0.055 & 0.051 & 0.067 & -0.041 \\
\hline & {$[0.015]^{* *}$} & {$[0.016]^{* *}$} & {$[0.027]^{*}$} & {$[0.076]$} \\
\hline \multirow[t]{2}{*}{ poverty_c==100-199\% } & -0.045 & -0.042 & -0.059 & 0.042 \\
\hline & {$[0.014]^{* *}$} & {$[0.016]^{* *}$} & {$[0.025]^{*}$} & {$[0.065]$} \\
\hline \multirow[t]{2}{*}{ poverty_c $==$ Less than $100 \%$} & -0.001 & -0.005 & 0.016 & -0.046 \\
\hline & {$[0.018]$} & {$[0.019]$} & {$[0.030]$} & {$[0.100]$} \\
\hline \multirow[t]{2}{*}{ unemployed_h } & 0.083 & 0.081 & 0.043 & -0.097 \\
\hline & {$[0.011]^{* *}$} & {$[0.012]^{* *}$} & {$[0.024]+$} & {$[0.055]+$} \\
\hline \multirow[t]{2}{*}{ married } & -0.012 & -0.016 & -0.002 & -0.058 \\
\hline & [0.012] & {$[0.014]$} & {$[0.021]$} & {$[0.068]$} \\
\hline \multirow[t]{2}{*}{ High school drop out Mother } & -0.048 & -0.056 & -0.066 & -0.065 \\
\hline & {$[0.022]^{*}$} & {$[0.025]^{*}$} & [0.035]+ & [0.094] \\
\hline \multirow[t]{2}{*}{ Some college education Mother } & 0.080 & 0.082 & 0.040 & 0.174 \\
\hline & {$[0.015]^{* *}$} & {$[0.016]^{* *}$} & {$[0.035]$} & {$[0.081]^{*}$} \\
\hline \multirow[t]{2}{*}{ College education degree Mother } & 0.097 & 0.090 & 0.132 & 0.075 \\
\hline & {$[0.015]^{* *}$} & {$[0.016]^{* *}$} & {$[0.031]^{* *}$} & {$[0.062]$} \\
\hline \multirow[t]{2}{*}{ Graduate School education Mother } & 0.132 & 0.130 & 0.093 & 0.155 \\
\hline & {$[0.021]^{* *}$} & {$[0.022]^{* *}$} & {$[0.048]+$} & {$[0.080]+$} \\
\hline \multirow[t]{2}{*}{ age_h } & -0.000 & 0.000 & 0.000 & 0.000 \\
\hline & {$[0.001]$} & {$[0.001]$} & {$[0.001]$} & {$[0.003]$} \\
\hline \multirow[t]{2}{*}{ nusualpl_h } & -0.056 & -0.052 & -0.094 & -0.141 \\
\hline & {$[0.015]^{* *}$} & {$[0.016]^{* *}$} & {$[0.032]^{* *}$} & {$[0.091]$} \\
\hline \multirow[t]{2}{*}{ nocoverage_h } & -0.094 & -0.104 & -0.062 & -0.243 \\
\hline & {$[0.018]^{* *}$} & {$[0.021]^{* *}$} & {$[0.024]^{*}$} & {$[0.077]^{* *}$} \\
\hline \multirow[t]{2}{*}{ goodhealth } & -0.411 & -0.442 & -0.316 & -0.532 \\
\hline & {$[0.026]^{* *}$} & {$[0.027]^{* *}$} & {$[0.048]^{* *}$} & {$[0.117]^{* *}$} \\
\hline \multirow[t]{2}{*}{ Survey year } & 0.003 & 0.001 & 0.010 & 0.007 \\
\hline & {$[0.003]$} & {$[0.003]$} & {$[0.006]+$} & {$[0.011]$} \\
\hline \multirow[t]{2}{*}{ Region of residence } & -0.032 & -0.031 & -0.033 & -0.034 \\
\hline & {$[0.006]^{* *}$} & {$[0.006]^{* *}$} & {$[0.010]^{* *}$} & {$[0.020]+$} \\
\hline \multirow[t]{2}{*}{ Constant } & -4.436 & -1.617 & -18.706 & -13.523 \\
\hline & {$[5.370]$} & [5.518] & [11.322]+ & [22.897] \\
\hline F statistic & 36.0 & 29.8 & 13.5 & 3.5 \\
\hline
\end{tabular}

$+p<0.1 ; * p<0.05 ; * * p<0.01$ 


\section{Percent Changes: Poisson - Individual models comparison}

\begin{tabular}{|c|c|c|c|c|c|}
\hline Variables & Native & Nat. Hispanic & Nat. Asian & Latin American & Asia \\
\hline \multirow[t]{2}{*}{ female_c } & 0.058 & 0.070 & 0.040 & 0.117 & 0.028 \\
\hline & {$[0.011]^{* *}$} & {$[0.034]^{*}$} & {$[0.108]$} & {$[0.039]^{* *}$} & {$[0.072]$} \\
\hline \multirow[t]{2}{*}{ Age } & 0.006 & -0.012 & -0.023 & -0.014 & 0.017 \\
\hline & {$[0.003]+$} & [0.011] & [0.031] & [0.011] & [0.024] \\
\hline \multirow[t]{2}{*}{ foreign_c } & -0.019 & 0.148 & -0.136 & -0.177 & -0.060 \\
\hline & {$[0.048]$} & [0.129] & {$[0.181]$} & {$[0.055]^{* *}$} & [0.112] \\
\hline \multirow[t]{2}{*}{ BMI mother } & -0.001 & 0.001 & -0.007 & -0.001 & 0.001 \\
\hline & {$[0.000]$} & {$[0.001]$} & {$[0.006]$} & {$[0.002]$} & [0.005] \\
\hline \multirow[t]{2}{*}{ obese } & 0.052 & 0.072 & 0.113 & 0.093 & -0.085 \\
\hline & {$[0.017]^{* *}$} & {$[0.045]$} & {$[0.165]$} & {$[0.052]+$} & [0.127] \\
\hline poverty_c $==100-199$ & {$[0.016]^{* *}$} & {$[0.039]^{*}$} & {$[0.128]$} & {$[0.048]$} & [0.105] \\
\hline poverty_c $==$ Less than 100 & {$[0.019]$} & {$[0.053]$} & {$[0.220]$} & {$[0.050]$} & [0.150] \\
\hline \multirow[t]{2}{*}{ unemployed_h } & 0.090 & 0.067 & 0.072 & 0.049 & -0.174 \\
\hline & {$[0.012]^{* *}$} & {$[0.037]+$} & [0.125] & {$[0.041]$} & {$[0.089]+$} \\
\hline \multirow[t]{2}{*}{ married } & -0.014 & -0.029 & 0.001 & 0.014 & -0.123 \\
\hline & {$[0.014]$} & [0.039] & [0.106] & [0.036] & [0.113] \\
\hline \multirow[t]{2}{*}{ High school drop out Mother } & -0.055 & -0.188 & 0.054 & -0.030 & -0.130 \\
\hline & {$[0.026]^{*}$} & {$[0.057]^{* *}$} & [0.195] & [0.057] & [0.148] \\
\hline \multirow[t]{2}{*}{ Some college education Mother } & 0.079 & 0.005 & 0.092 & 0.097 & 0.259 \\
\hline & {$[0.016]^{* *}$} & {$[0.053]$} & [0.182] & [0.061] & {$[0.125]^{*}$} \\
\hline \multirow[t]{2}{*}{ College education degree Mother } & 0.092 & 0.094 & 0.195 & 0.240 & 0.069 \\
\hline & {$[0.016]^{* *}$} & {$[0.045]^{*}$} & [0.155] & {$[0.061]^{* *}$} & [0.095] \\
\hline \multirow[t]{2}{*}{ Graduate School education Mother } & 0.129 & 0.039 & 0.265 & 0.209 & 0.189 \\
\hline & {$[0.022]^{* *}$} & [0.073] & [0.203] & {$[0.100]^{*}$} & [0.119] \\
\hline \multirow[t]{2}{*}{ age_h } & 0.000 & 0.003 & -0.001 & -0.002 & -0.001 \\
\hline & {$[0.001]$} & {$[0.002]$} & {$[0.006]$} & {$[0.003]$} & [0.005] \\
\hline \multirow[t]{2}{*}{ nusualpl_h } & -0.048 & -0.065 & -0.228 & -0.153 & -0.160 \\
\hline & {$[0.016]^{* *}$} & {$[0.049]$} & {$[0.207]$} & {$[0.057]^{* *}$} & [0.148] \\
\hline \multirow[t]{2}{*}{ nocoverage_h } & -0.100 & -0.062 & -0.146 & -0.098 & -0.337 \\
\hline & {$[0.022]^{* *}$} & [0.045] & {$[0.240]$} & {$[0.039]^{*}$} & {$[0.126]^{*}$} \\
\hline \multirow[t]{2}{*}{ goodhealth } & -0.441 & -0.438 & -0.831 & -0.366 & -0.723 \\
\hline & {$[0.027]^{* *}$} & {$[0.066]^{* *}$} & {$[0.206]^{* *}$} & {$[0.095]^{* *}$} & {$[0.216]^{* *}$} \\
\hline \multirow[t]{2}{*}{ Survey year } & 0.001 & -0.001 & -0.006 & 0.023 & 0.019 \\
\hline & {$[0.003]$} & {$[0.008]$} & {$[0.026]$} & {$[0.010]^{*}$} & [0.018] \\
\hline \multirow[t]{2}{*}{ Region of residence } & -0.031 & -0.033 & -0.044 & -0.053 & -0.035 \\
\hline & {$[0.006]^{* *}$} & {$[0.016]^{*}$} & [0.047] & {$[0.018]^{* *}$} & [0.031] \\
\hline \multirow[t]{2}{*}{ yrsinusrec_h $\mathrm{h}==1.0000$} & & & & -0.209 & 0.141 \\
\hline & & & & {$[0.114]+$} & [0.177] \\
\hline \multirow[t]{2}{*}{ yrsinusrec $\_h==2.0000$} & & & & -0.195 & -0.129 \\
\hline & & & & {$[0.093]^{*}$} & {$[0.170]$} \\
\hline \multirow[t]{2}{*}{ Constant } & -0.766 & 3.708 & 13.214 & & \\
\hline & [5.699] & [16.264] & [51.520] & & \\
\hline
\end{tabular}

$+p<0.1 ; * p<0.05 ; * * 00.01$ 